\title{
AN APPLICATION OF THE ALGEBRA OF DIFFERENTIALS OF INFINITE RANK
}

\author{
WILLIAM C. BROWN
}

\begin{abstract}
Let $k$ denote an arbitrary field and let $R$ be an affine local domain over $k$. Let $\left(\Omega_{k}(R), \delta_{k}^{n}\right)$ be the universal algebra of $k$-higher differentials over $R$. Let $K$ be the quotient field of $R$ and $L$ the residue class field of $R$. If $K$ is a separable extension of $k$ and $L$ is a separable algebraic extension of $k$, then it is shown that $R$ is a regular local ring if and only if $\Omega_{k}(R)$ is a free $R$-algebra. If both $K$ and $L$ are separable extensions of $k$ and $R$ has a separating residue class field, then $R$ is a regular local ring if and only if $\Omega_{k}(R)$ is a free $R$-algebra.
\end{abstract}

Introduction. Let $k$ and $A$ denote commutative associative rings with identities. We assume throughout this paper that $A$ is a $k$-algebra. Thus we have a ring homomorphism $\theta: k \rightarrow A$ which sends the identity of $k$ to the identity of $A$. If $x$ is an element of $k$ and $b$ an element of $A$, we shall denote $0(x) b$ by just $x b$. By an $A$-algebra $V$, we shall mean a commutative and associative ring $V$ which is a unitary $A$-module, and for all $v_{1}, v_{2}$ in $V$ and $a$ in $A$ we have $a\left(v_{1} v_{2}\right)=\left(a v_{1}\right) v_{2}=v_{1}\left(a v_{2}\right)$.

A $k$-higher derivation $\delta=\left\{\delta_{i}\right\}$ of $A$ into $V$ is an infinite sequence $\delta_{1}, \delta_{2}, \delta_{3}, \cdots$ of maps $\delta_{i}: A \rightarrow V$ such that

(a) each $\delta_{i}$ is an element of $\operatorname{Hom}_{k}(A, V)$;

(b) for all $a$ and $b$ in $A$ and $i \geqq 1$, we have

$$
\delta_{i}(a b)=a \delta_{i}(b)+\delta_{1}(a) \delta_{i \cdots 1}(b)+\cdots+\delta_{i-1}(a) \delta_{1}(b)+\delta_{i}(a) b .
$$

We shall abbreviate this last line by writing

$$
\delta_{i}(a b)=\sum_{j+k=i} \delta_{j}(a) \delta_{k}(b) .
$$

In [2], the author and W. E. Kuan used this notion of $k$-higher derivations to obtain some new results on analytic products of a variety $V$ along a subvariety $W$. In this note, we shall show that higher derivations can be used to study simple points of algebraic varieties.

Received by the editors November 9,1971 .

AMS 1970 subject classifications. Primary 13H05.

Key words and phrases. $k$-higher derivations, algebra of $k$-higher differentials, separating residue class field, separating representatives. 
In $[1]^{1}$, the present author introduced the universal algebra $\Omega_{k}(A)$ of higher differentials on $A$ and explored the functorial properties of $\Omega_{k}($ ). $\Omega_{k}(A)$ is an $A$-algebra (without identity) which has the following properties:

(a) There exists a canonical $k$-higher derivation $\delta_{k}^{A}=\left\{\delta_{k i}^{A}\right\}$ of $A$ into $\Omega_{k}(A)$.

(b) $\Omega_{k}(A)$ is generated as an $A$-algebra by the set $\left\{\delta_{k i}^{A}(a) \mid a \in A, i \geqq 1\right\}$.

(c) $\left(\Omega_{k}(A), \delta_{k}^{A}\right)$ has the following universal mapping property: If $V$ is any $A$-algebra and $\lambda=\left\{\lambda_{i}\right\}$ a $k$-higher derivation of $A$ into $V$, then there exists a unique $A$-algebra homomorphism $\psi: \Omega_{k}(A) \rightarrow V$ such that for all $i \geqq 1$ we have $\lambda_{i}=\psi \delta_{k i}^{A}$.

The construction of $\Omega_{k}(A)$ is briefly as follows: Let $I$ be the kernel of the multiplication map $P: A \otimes_{k} A \rightarrow A$ given by $P\left(\sum x_{j} \otimes y_{j}\right)=\sum x_{j} y_{j}$. Let $I^{\prime}$ be the direct sum of the $A$-modules $I / I^{i+1}$ for $i \geqq 1$. Thus

$$
I^{\prime}=\sum_{i \geqq 1} \oplus I / I^{i+1} \text {. }
$$

We now form the symmetric algebra $S\left(I^{\prime}\right)$ of $I^{\prime}$ over $A$ but without terms of degree zero. Thus

$$
S\left(I^{\prime}\right)=I^{\prime} \oplus\left\{\frac{I^{\prime} \otimes_{A} I^{\prime}}{B_{2}}\right\} \oplus\left\{\frac{I^{\prime} \otimes_{A} I^{\prime} \otimes_{A} I^{\prime}}{B_{3}}\right\} \oplus \cdots
$$

where $B_{n}$ is the $A$-submodule of $I^{\prime} \otimes_{A} I^{\prime} \otimes_{A} \cdots \otimes_{A} I^{\prime}$ ( $n$ factors) generated by all elements of the form $s_{1} \otimes_{A} \cdots \otimes_{A} s_{n}-s_{\mathfrak{G}(1)} \otimes_{A} \cdots \otimes_{A} s_{\mathfrak{G}(n)}$. Here $s_{1}, \cdots, s_{n}$ are elements of $I^{\prime}$ and $\mathfrak{G}_{\mathfrak{S}}$ is any permutation of $\{1, \cdots, n\}$. The mapping $\delta^{i}: A \rightarrow I / I^{i+1}$ defined by

$$
\delta^{i}(a)=\left(1 \otimes_{k} a-a \otimes_{k} 1\right)+I^{i+1}
$$

is an $i$ th order $k$-derivation of $A$ into $I / I^{i+1}$ [5]. Thus each $\delta^{i}$ induces an $i$ th order $k$-derivation of $A$ into $S\left(I^{\prime}\right)$. Let $J$ denote the ideal in $S\left(I^{\prime}\right)$ which is generated by all elements of the form

$$
\delta^{i}(a b)-\sum_{j+k=i} \delta^{j}(a) \delta^{k}(b), \quad a, b \text { in } A, i \geqq 1 .
$$

Then $S\left(I^{\prime}\right) / J=\Omega_{k}(A)$ and $\delta_{k i}^{A}$ is the induced map from $\delta^{i}$. One can now show that $\left(\Omega_{k}(A), \delta_{k}^{A}\right)$ satisfy conditions $a, b$ and $c$ [1, Theorem 1].

In this paper, we shall show that under suitable conditions the simplicity of a point on a variety defined over a field $k$ can be determined by studying $\Omega_{k}(R), R$ the local ring at the point in question.

\footnotetext{
${ }^{1}$ It has recently come to my attention that $\mathrm{P}$. Ribenboim in Higher derivations of rings. I (Rev. Roumaine Math. Pures Appl., Tome XVI, Bucarest, 1971, pp. 77-110) has also constructed a universal object using different techniques than those in [1].
} 
Main results. Let $\left(\Omega_{k}^{1}(A), \delta_{A / k}^{1}\right)$ denote the universal object for $k$ derivations of rank one (ordinary derivations) on $A$ ([4] or [5]). We need the following important lemma:

Lemma. $\quad \Omega_{k}^{1}(A)$ is an $A$-module direct summand of $\Omega_{k}(A)$.

Proof. It is well known $\left[5\right.$, p. 15] that $\Omega_{k}^{1}(A)=I / I^{2}$ and $\delta_{A / k}^{1}(a)=$ $\left(1 \otimes_{k} a-a \otimes_{k} 1\right)+I^{2}$. We note that $I / I^{2}$ is an $A$-algebra in which the product of any two elements is zero. This allows us to extend $\delta_{A / k}^{1}$ to a $k$-higher derivation $\delta=\left\{\delta_{i}\right\}: A \rightarrow \Omega_{k}^{1}(A)$ in the following way: For each $i \geqq 1$, define $\delta_{i}=\delta_{A / k}^{1}$. Then each $\delta_{i}$ is a $k$-linear mapping of $A$ into $\Omega_{k}^{1}(A)$ and, for all $i \geqq 1$,

$$
\delta_{i}(a b)=\sum_{j+i=k} \delta_{j}(a) \delta_{k}(b) .
$$

Now from the universal mapping property of $\left(\Omega_{k}(A), \delta_{k}^{A}\right)$, there exists a unique $A$-algebra homomorphism $\psi_{1}: \Omega_{k}(A) \rightarrow \Omega_{k}^{1}(A)$ such that for all $i \geqq 1$, $\psi_{1} \delta_{k i}^{A}=\delta_{i}=\delta_{A / k}^{1}$. From the universal mapping property of $\left(\Omega_{k}^{1}(A), \delta_{A / k}^{1}\right)$, there exists a unique $A$-module homomorphism $\psi_{2}: \Omega_{k}^{1}(A) \rightarrow \Omega_{k}(A)$ such that $\psi_{2} \delta_{A / k}^{1}=\delta_{k 1}^{A}$. It now easily follows that $\psi_{1} \psi_{2}$ is the identity on $\Omega_{k}^{1}(A)$. Thus $\Omega_{k}^{1}(A)$ is an $A$-module direct summand of $\Omega_{k}(A)$.

Throughout the rest of this paper, $k$ will denote an arbitrary field. Suppose $K$ is a field which contains $k$. If $\left\{u_{\alpha} ; \alpha \in \Lambda\right\}$ is a set of indeterminates over $K$, then we shall denote by $K\left\langle u_{\alpha} \mid \alpha \in \Lambda\right\rangle$ the commutative ring of polynomials in the $u_{\alpha}$, coefficients in $K$, which have no constant term. Thus if $K\left[u_{\alpha} \mid \alpha \in \Lambda\right]$ denotes the ordinary ring of polynomials, $K\left\langle u_{\boldsymbol{x}}\right\rangle+K=$ $K\left[u_{\alpha}\right]$. We shall need the following theorem:

THEOREM 1. Let $K$ be a separable extension of $k$. Suppose the transcendence degree of $K$ over $k$ is $n \geqq 1$. Let $\left\{u_{i j} \mid j=1, \cdots, n, i=1, \cdots, \infty\right\}$ be a collection of indeterminates over $K$. Then

$$
\Omega_{k}(K) \cong K\left\langle u_{i j}\right| j=1, \cdots, n, i=1, \cdots, \infty \text {; }
$$

Proof. Let $x_{1}, \cdots, x_{n}$ be elements of $K$ which are algebraically independent over $k$ and such that $K$ is a separable algebraic extension of $k\left(x_{1}, \cdots, x_{n}\right)$. Now it is known that $\Omega_{k}\left(k\left[x_{1}, \cdots, x_{n}\right]\right) \cong k\left[x_{1}, \cdots, x_{n}\right] \times$ $\left\langle u_{i j}\right\rangle$ [1, p. 29]. It follows from [1, Theorem 6] that $\Omega_{k}\left(k\left(x_{1}, \cdots, x_{n}\right)\right) \cong$ $k\left(x_{1}, \cdots, x_{n}\right)\left(u_{i j}\right\rangle$. Let $S=k\left(x_{1}, \cdots, x_{n}\right)$. Then the canonical $k$-higher derivation $\delta_{k}^{s}$ of $S$ into $\Omega_{k}(S)$ is given by $\delta_{k i}^{s}\left(x_{j}\right)=u_{i j}$.

Now we may view $\delta_{k}^{s}$ as a $k$-higher derivation of $S$ into $Q\left(K\left\langle u_{i j}\right\rangle\right)$, the quotient field of $K\left\langle u_{i j}\right\rangle$. By using Zorn's lemma and [3, Proposition 2], we may uniquely extend $\delta_{k}^{s}$ to a $k$-higher derivation $\hat{\delta}$ of $K$ into $Q\left(K\left\langle u_{i j}\right\rangle\right)$. Since $K$ is a separable algebraic extension of $S$, one can easily argue that, for all $q, \hat{\delta}_{q}(K) \subset K\left\langle u_{i j}\right\rangle$. Thus $\hat{\delta}$ is a $k$-higher derivation of $K$ into $K\left\langle u_{i j}\right\rangle$. 
It now follows easily that $\left(K\left\langle u_{i j}\right\rangle, \hat{\delta}\right)$ has the universal mapping property (c). Hence it follows that $\Omega_{k}(K) \cong K\left\langle u_{i j}\right\rangle$.

By an affine ring over $k$, we shall mean any homomorphic image of $k\left[X_{1}, \cdots, X_{m}\right]$, the polynomial ring in a finite number of indeterminates over $k$. Let $A$ be a $k$-algebra. We shall say that $\Omega_{k}(A)$ is a free $A$-algebra if $\Omega_{k}(A)$ is isomorphic as an $A$-algebra to $A\left\langle X_{\alpha} ; \alpha \in \Lambda\right\rangle$. Here the $\left\{X_{\alpha} ; \alpha \in \Lambda\right\}$ is a collection of indeterminates indexed by some set $\Lambda$ and $A\left\langle X_{\alpha} ; \alpha \in \Lambda\right\rangle$ denotes the collection of all polynomials in the $X_{\alpha}$, coefficients in $A$, which have no constant term. We note that if $\Omega_{k}(A)$ is a free $A$-algebra then $\Omega_{k}(A)$ is a free $A$-module. Hence if $A$ is a local ring, the lemma implies that $\Omega_{k}^{1}(A)$ is a free $A$-module if $\Omega_{k}(A)$ is a free $A$-algebra.

THEOREM 2. Let $A$ be an affine ring over $k$ and let $R$ be the quotient ring of $A$ with respect to a prime ideal $p$. Assume $\left(1^{\circ}\right) A$ is an integral domain; $\left(2^{\circ}\right)$ the quotient field $K$ of $A$ is a separable extension of $k ;\left(3^{\circ}\right)$ the residue class field $L$ of $R$ is a separable algebraic extension of $k$. Then $R$ is a regular local ring if and only if $\Omega_{k}(R)$ is a free $R$-algebra.

Proof. Let us first suppose that $R$ is a regular local ring. Let $\left\{z_{1}, \cdots, z_{n}\right\}$ be any regular system of parameters of $R$. Note that the hypotheses of Theorem 2 imply that $p$ is a maximal ideal of $A$. Hence $n=\operatorname{dim} R$ is equal to the transcendence degree of $K$ over $k$. Further it is known that $\left\{z_{1}, \cdots, z_{n}\right\}$ is a separating transcendence basis of $K$ over $k$ [4, Theorem 3, Corollary 2]. Since $K$ is a separable algebraic extension of $k\left(z_{1}, \cdots, z_{n}\right)$, Theorem 1 implies $\Omega_{k}(K)=K\left\langle u_{i j} \mid j=1, \cdots, n, i=1, \cdots, \infty\right\rangle$ for indeterminates $u_{i j}$ over $K$. Further we know that $u_{i j}=\delta_{k i}^{K}\left(z_{j}\right)$. Now $K \otimes_{R} \Omega_{k}(R) \cong \Omega_{k}(K)$ [1, Theorem 6] under the $K$-algebra mapping sending

$$
1 \otimes_{R} \delta_{k i}^{R}(x) \rightarrow \delta_{k i}^{K}(x) \text { for } x \text { in } R .
$$

Thus it is clear that $\left\{\delta_{k i}^{R}\left(z_{j}\right) \mid j=1, \cdots, n, i=1, \cdots, \infty\right\}$ is a collection of free elements in $\Omega_{k}(R)$. That is, a polynomial $f$ in the $\delta_{k i}^{R}\left(z_{j}\right)$ with coefficients in $R$ is zero if and only if each of the coefficients of $f$ is zero. Hence $\left\{\delta_{k i}^{R}\left(z_{j}\right)\right\}$ forms a free basis for $\Omega_{k}(R)$ if they generate all of $\Omega_{k}(R)$ as an $R$-algebra.

So let $C=R\left\langle\delta_{k i}^{R}\left(z_{j}\right) ; j=1, \cdots, n, i=1, \cdots, \infty\right\rangle$. Then $C$ is the $R$ subalgebra of $\Omega_{k}(R)$ generated by the set $\left\{\delta_{k i}^{R}\left(z_{j}\right)\right\}$. We wish to show that $C=\Omega_{k}(R)$.

Let $\left(\Omega_{k}^{1}(R), \delta_{R / k}^{1}\right)$ denote the universal object for $k$-derivations of rank one ([4] or [5]). It follows from the proof of Theorem 3 in [4] that $\Omega_{k}^{1}(R)$ is generated as an $R$-module by $\delta_{R / k}^{1}\left(z_{1}\right), \cdots, \delta_{R / k}^{1}\left(z_{n}\right)$. Now consider $\delta_{k 1}^{R}: R \rightarrow \Omega_{k}(R)$. Since $\delta_{k 1}^{R}$ is a derivation of $R$ into $\Omega_{k}(R)$, there exists a unique $R$-module homomorphism $\psi: \Omega_{k}^{1}(R) \rightarrow \Omega_{k}(R)$ such that $\psi \delta_{R / k}^{1}=\delta_{k 1}^{R}$. 
Now let $x$ be an element of $R$. Then there exists $r_{1}, \cdots, r_{n}$ in $R$ such that $\delta_{R / k}^{1}(x)=\sum r_{i} \delta_{R / k}^{1}\left(z_{i}\right)$. Thus $\delta_{k 1}^{R}(x)=\psi \delta_{R / k}^{1}(x)=\sum r_{i} \psi \delta_{R k_{k}}^{1}\left(z_{\imath}\right)=\sum r_{i} \delta_{k 1}^{R}\left(z_{i}\right)$. Thus the image of $\delta_{k 1}^{R}$ is contained in $C$.

Assume we have shown that the images of $\delta_{k 1}^{R}, \cdots . \delta_{k m}^{R}$ are contained in $C$. Consider $\delta_{k, m+1}^{R}: R \rightarrow \Omega_{k}(R)$. Then $\delta_{k, m+1}^{R}$ induces a derivation $\delta$ of rank one from $R$ to the $R$-module $\Omega_{k}(R) / C$ in the following way:

$$
\delta(x)=\delta_{k, m+1}^{R}(x)+C, \quad x \text { in } R .
$$

Clearly $\delta$ is a $k$-linear homomorphism of $R$ into $\Omega_{k}(R) / C$. Let $x$ and $y$ be elements of $R$. Then we have

$$
\begin{aligned}
\delta(x y) & =\delta_{k, m+1}^{R}(x y)+C=\left(\sum_{i+j=m+1} \delta_{k i}^{R}(x) \delta_{k j}^{R}(y)\right)+C \\
& =\left\{x \delta_{k, m+1}^{R}(y)+y \delta_{k, m+1}^{R}(x)+\sum_{i+j=m+1 ; i, j>0} \delta_{k i}^{R}(x) \delta_{k j}^{R}(y)\right\}+C .
\end{aligned}
$$

Now by the induction hypothesis,

Thus

$$
\sum_{i+j=m+1 ; i, j>0} \delta_{k i}^{R}(x) \delta_{k j}^{R}(y) \in C .
$$

$$
\begin{aligned}
\delta(x y) & =x\left\{\delta_{k, m+1}^{R}(y)+C\right\}+y\left\{\delta_{k, m+1}^{R}(x)+C^{\prime}\right. \\
& =x \delta(y)+y \delta(x) .
\end{aligned}
$$

Hence $\delta$ is a derivation of $R$ into $\Omega_{k}(R) / C$. From the universal mapping property of $\left(\Omega_{k}^{1}(R), \delta_{R / k}^{1}\right)$, there exists a unique $R$-module homomorphism $\psi_{1}: \Omega_{k}^{1}(R) \rightarrow \Omega_{k}(R) / C$ such that $\delta(x)=\psi_{1} \delta_{R / k}^{1}(x)$. Thus the image of $\delta$ is generated by $\delta\left(z_{1}\right), \cdots, \delta\left(z_{n}\right)$. This immediately implies that the image of $\delta_{k, m+1}^{R}$ is contained in $C$. Thus $C$ contains $\left\{\delta_{k i}^{R}(x) \mid x \in R, i \geqq 1\right\}$. Since $\Omega_{k}(R)$ is generated as an $R$-algebra by this set, we get $C=\Omega_{k}(R)$.

Thus if $R$ is a regular local ring with regular system of parameters $\left\{z_{1}, \cdots, z_{n}\right\}$ then $\Omega_{k}(R)$ is a free $R$-algebra with basis $\left\{\delta_{k i}^{R}\left(z_{j}\right)\right\}$.

The converse follows immediately from the lemma and [4, Theorem 3].

Since an irreducible subvariety $W$ of an irreducible variety $V$ is simple if and only if the local ring $Q(W / V)$ of $W$ on $V$ is regular, we can rephrase Theorem 2 as follows:

Corollary. Let $V$ be an irreducible affine variety defined over $k$. Let $P$ be a point of $V$ such that $k(P)$ is a separable algebraic extension of $k$. Then $P$ is a simple point of $V$ if and only if $\Omega_{k}(Q(P / V))$ is a free $Q(P / V)$-algebra. Here of course $Q(P / V)$ denotes the local ring of $P$ at $V$. 
As Y. Nakai has shown in [4], if either hypothesis $\left(2^{\circ}\right)$ or $\left(3^{\circ}\right)$ is dropped, $\Omega_{k}^{1}(R)$ being a free $R$-module is no longer equivalent to $R$ being a regular local ring. Hence the hypotheses $\left(2^{\circ}\right)$ and $\left(3^{\circ}\right)$ in Theorem 2 are inevitable.

If $R$ has a separating residue class field $L$, then we can generalize Theorem 2 .

Let $R$ be a local integral domain containing the field $k$ and having quotient field $K$. Let $L$ be the residue class field of $R$ and assume $L$ is a separable extension of $k$. Let $\left\{u_{1}, \cdots, u_{s}\right\}$ be a separating transcendence basis of $L / k$. Then we can find representatives $\alpha_{1}, \cdots, \alpha_{s}$ of $u_{1}, \cdots, u_{s}$ in $R$. The elements $\alpha_{1}, \cdots, \alpha_{s}$ are clearly algebraically independent over $k$ and $F=k\left(\alpha_{1}, \cdots, \alpha_{s}\right)$ is a subfield of $R$. If it is possible to find $u_{i}$ 's and $\alpha_{i}$ 's as above such that $K$ is a separable extension of $F$, then we say $R$ has a separating residue class field. If $R$ has a separating residue class field, we shall call $\alpha_{1}, \cdots, \alpha_{s}$ separating representatives in $R$.

THEOREM 3. Let $A$ be an affine ring over the field $k$ and let $R$ be the quotient ring of $A$ with respect to a prime ideal $p$ in $A$. Assume $\left(1^{\circ}\right) A$ is an integral domain, $\left(2^{\circ}\right)$ the quotient field $K$ of $R$ is a separable extension of $k$, $\left(3^{\circ}\right)$ the residue class field $L$ of $R$ is a separable extension of $k,\left(4^{\circ}\right) R$ has a separating residue class field. Then $R$ is a regular local ring if and only if $\Omega_{k}(R)$ is a free $R$-algebra.

Proof. Let us first assume $R$ is a regular local ring. Let $\left\{z_{1}, \cdots, z_{n}\right\}$ be a regular system of parameters for $R$ and $\left\{\alpha_{1}, \cdots, \alpha_{s}\right\}$ separating representatives in $R$. Since the quotient field of $A / p$ is $L$, we know the transcendence degree of $K / k$ is exactly equal to $n+s$. $K$ is a separable extension of $F=k\left(\alpha_{1}, \cdots, \alpha_{s}\right)$ and as pointed out in the proof of Theorem $2,\left\{z_{1}, \cdots, z_{n}\right\}$ is a separating transcendence basis of $K / F$. Hence $\left\{z_{1}, \cdots, z_{n}, \alpha_{1}, \cdots, \alpha_{s}\right\}$ is a separating transcendence basis for $K / k$. Thus it follows from Theorem 1 that $\left\{\delta_{k i}^{K}\left(z_{j}\right), \delta_{k i}^{K}\left(\alpha_{l}\right) \mid j=1, \cdots, n\right.$, $l=1, \cdots, s, i=1, \cdots, \infty\}$ forms a free $K$-algebra basis for $\Omega_{k}(K)$. Now $\Omega_{k}(K) \cong K \otimes_{R} \Omega_{k}(R)$. Hence as in Theorem $2,\left\{\delta_{k i}^{R}\left(z_{j}\right), \delta_{k i}^{R}\left(\alpha_{l}\right)\right\}$ will form a free $R$-algebra basis for $\Omega_{k}(R)$ if they generate all of $\Omega_{k}(R)$.

Let $C$ be the $R$-subalgebra of $\Omega_{k}(R)$ generated by $\left\{\delta_{k i}^{R}\left(z_{j}\right), \delta_{k i}^{R}\left(\alpha_{l}\right)\right\}$. We shall first show that the image of $\delta_{k 1}^{R}: R \rightarrow \Omega_{k}(R)$ is continued in $C$. As before, let $\Omega_{k}^{1}(R)$ denote the universal object for $k$-derivations of rank one on $R$. Then it follows from [4, Proposition 3] that

$$
\text { * }: 0 \longrightarrow R \otimes_{F} \Omega_{k}^{1}(F) \stackrel{\mu}{\longrightarrow} \Omega_{k}^{1}(R) \stackrel{\alpha}{\longrightarrow} \Omega_{F}^{1}(R) \longrightarrow 0
$$

is an exact sequence of $R$-modules. Here $\mu$ is the $R$-module homomorphism given by $\mu\left(1 \odot \delta_{F / k}^{1}(x)\right)=\delta_{R / k}^{1}(x)$ and $\alpha$ can be taken to be the $R$-module homomorphism which sends $\delta_{R / k}^{1}(x)$ to $\delta_{R / F}^{1}(x)$. 
Now by [4, Theorem 3], $\Omega_{F}^{1}(R)$ is a free $R$-module generated by $\delta_{R / F}^{1}\left(z_{1}\right), \cdots, \lambda_{R / F}^{1}\left(z_{n}\right) . \Omega_{k}^{1}(F)$ is a free $F$-module generated by $\delta_{F / k}^{1}\left(\alpha_{1}\right)$, $\cdots, \delta_{F / k}^{1}\left(\alpha_{s}\right)$. Hence it follows that $\Omega_{k}^{1}(R)$ is a free $R$-module with basis $\delta_{R / k}^{1}\left(z_{1}\right), \cdots, \delta_{R / k}^{1}\left(z_{n}\right), \delta_{R / k}^{1}\left(\alpha_{1}\right), \cdots, \delta_{R / k}^{1}\left(\alpha_{s}\right)$.

From the universal mapping property of $\left(\Omega_{k}^{1}(R), \delta_{R / k}^{1}\right)$ we get a unique $R$-module homomorphism $\psi: \Omega_{k}^{1}(R) \rightarrow \Omega_{k}(R)$ such that for all $x$ in $R, \delta_{k 1}^{R}(x)=$ $\psi \delta_{R / k}^{1}(x)$. As in the proof of Theorem 2, we get that the image of $\delta_{k 1}^{R}$ is contained in $\sum R \delta_{k 1}^{R}\left(z_{j}\right)+\sum R \delta_{k 1}^{R}\left(\alpha_{l}\right)$ which in turn is contained in $C$. The proof now follows as in Theorem 2 and we get $C=\Omega_{k}(R)$. Thus if $R$ is a reguiar local ring, and $\left\{z_{1}, \cdots, z_{n}\right\}$ is any regular system of parameters of $R$, then $\left\{\delta_{k i}^{R}\left(z_{j}\right), \delta_{k i}^{R}\left(\alpha_{l}\right)\right\}$ forms a free $R$-algebra basis for $\Omega_{k}(R)$.

As in Theorem 2, the converse follows from the lemma and [4, Theorem $\left.3^{\prime}\right]$.

COROLlaRY. Let $V$ be an irreducible affine variety defined over the field $k$. Let $P$ be a point of $V$ (not necessarily algebraic over $k$ ). Assume $Q(P / V)$ has a separating residue class field. Then $P$ is a simple point of $V$ if and only if $\Omega_{k}(Q(P / V))$ is a free $Q(P / V)$-algebra.

\section{REFERENCES}

1. W. C. Brown, The algebra of differentials of infinite rank (to appear).

2. W. C. Brown and W. E. Kuan, Ideals and higher derivations in commutative rings, Canad. J. Math. (to appear).

3. N. Heerema, Higher derivations and automorphisms of complete local rings, Bull. Amer. Math. Soc. 76 (1970), 1212-1225, MR 42 \#1818.

4. Y. Nakai, On the theory of differentials in commutative rings, J. Math. Soc. Japan 13 (1961), 63-84. MR 23 \#A2437.

5. ——, Higher order derivations. I, Osaka J. Math. 7 (1970), 1-27. MR 41 \#8404.

Department of Mathematics, Michigan State University, East lansing, MICHIGAN 48823 